\title{
Decoding the life story of our epigenome
}

\author{
Anthony S Zannas*,1,2,3,4 \\ ${ }^{1}$ Department of Psychiatry, University of North Carolina, Chapel Hill, NC, USA \\ ${ }^{2}$ Department of Genetics, University of North Carolina, Chapel Hill, NC, USA \\ ${ }^{3}$ Department of Psychiatry \& Behavioral Sciences, Duke University Medical Center, Durham, NC, USA \\ ${ }^{4}$ Institute for Trauma Recovery, University of North Carolina, Chapel Hill, NC, USA \\ *Author for correspondence: anthony_zannas@med.unc.edu
}

\section{"we now know of a biological process that may explain how, as they had all believed, our lives shape us; and this process is epigenetics."}

First draft submitted: 2 June 2019; Accepted for publication: 1 July 2019; Published online: 6 September 2019

Ever since antiquity, it has been recognized that our life stories - the chosen or unchosen encounters and ways in which we live - constantly shape us. To quote two ancient Greek philosophers, Heraclitus believed that "Day by day, what we choose, think, and do is who we become" and Aristotle that "habit is second nature." Several centuries later, Hans Selye, the harbinger of modern stress research, viewed every environmental demand as a stressor that leaves an 'indelible scar' on our bodies. The resounding theme across these prominent thinkers is that what happens in our lives can change us, for better or for worse.

Over the last decades, a vast body of research has grounded these long-standing ideas on quantitative evidence, showing that life encounters, behaviors, and stressors can profoundly influence lifespan and health span. Highly prevalent lifestyle parameters and behaviors - smoking, unhealthy diets, sedentary lifestyle and alcohol - are key risk factors for a host of disease states - including cardiovascular diseases, cancer, diabetes, and chronic respiratory syndromes - together responsible for $70 \%$ of deaths worldwide [1]. Chronic psychosocial stress - a hallmark of modern, fast-paced societies - not only predisposes to health-damaging behaviors but also directly affects our body physiology, contributing to negative outcomes [2]. Yet the impact of these risk factors varies broadly across individuals, and gaining insights into how life stories shape individual health outcomes can contribute to bettertargeted, personalized interventions.

But what can we do to gain such insights? And, in particular, how can we accomplish that today, in the 'molecular age'? We could imagine a scenario where Heraclitus, Aristotle, or Selye, seeking answers to these questions, meet a molecular biologist in a modern coffee shop to get an update on recent scientific advances. Such an imaginary exchange would make them exclaim with enthusiasm: 'We knew it.' For we now know of a biological process that may explain how, as they had all believed, our lives shape us; and this process is epigenetics.

\section{Excitement \& skepticism}

There is perhaps no more intuitive term to describe epigenetics than the Aristotelian 'second nature'. Epigenetics - a composite Greek word derived from the prefix 'epi', meaning 'upon', and the word 'genetics' - encompasses the ever-growing repertoire of biological processes that regulate chromatin structure and gene function without changing the underlying genetic code. These processes can be conceptualized as molecular switches that turn genes on and off, or, better still, as rheostats that fine-tune gene activity. In many cases, epigenetic modifications last long and influence cell and body function, thereby acting 'on top' of our genetic makeup to change our very nature. But what do we know about how life encounters may shape the epigenetic landscape, and how could we someday use this knowledge to promote health?

A major breakthrough has been the development of microarray technology able to measure DNA methylation, one of the critical epigenetic modifications, at hundreds of thousands of individual sites scattered across the human genome. By employing this technology in human populations, scientists have begun to witness composite epigenetic patterns associated with different aspects of human life. Such composite DNA methylation markers have reached particularly high accuracy for predicting chronological age and show reproducible associations with aging-related 
disease outcomes [3], but predictive scores derived from multiple sites also exist for smoking [4] and alcohol use [5]. Genome-wide DNA methylation analyses also suggest that distinct epigenetic sites may be particularly susceptible to psychosocial stress [6]. These observations raise the possibility that one day we might be able to predict how individual life experiences may 'get under our skin' by becoming embedded as distinct epigenetic signatures.

Such predictive ability would have enormous implications for several reasons. First, using genome-wide screens to chart the epigenetic sites regulated by each environmental stressor can point to yet unknown genes and their products that, when gone awry, can contribute to disease processes. Uncovering these molecular culprits could open novel avenues for intervention. Second, epigenetic signatures can vary among individuals with similar life environments and genetic makeup [7]. Tracking these changes early after risk exposure may enable us to predict potential disease processes as they emerge in vulnerable individuals. Third, unlike genetic mutations, epigenetic changes are preventable and even reversible. Epigenetic drugs able to manipulate or reset these changes are a promising treatment strategy that has improved disease trajectories in certain types of cancer [8].

Despite these exciting prospects, epigenetics is currently met with skepticism due to several challenges and limitations. As mentioned above, array technology-based studies in human populations have identified an expanding number of epigenetic changes associated with environmental stressors; however, the mechanisms underlying these associations and the temporal cascade of molecular events remain elusive. This lack of molecular insights impedes the development of reliable biomarkers and targeted interventions. Besides a few exceptions, such as in some cancers where epigenetic markers have begun to categorize patients in prognostic groups [8], epigenetic changes currently appear in large part stochastic, having limited value as disease biomarkers. Another impediment in uncovering reliable epigenetic signatures is their cell and tissue specificity. Unlike genetic sequence, each body tissue has a distinct epigenetic landscape that reflects its specific function, and to date most studies examining associations with life environment have used easily accessible tissues, such as blood or saliva, instead of those directly relevant for the disease of interest. Together these limitations create a gap between our preclinical insights into epigenetic processes and their translation to clinical applications.

\section{What needs to be done}

The quest to unravel how our life stories shape our epigenomes to contribute to health outcomes will undoubtedly be a formidable one, requiring the orchestrated efforts of scientists and policy makers along with public engagement. These efforts can be systematized across four axes: establishing large-scale, longitudinal studies; examining both negative and positive life environments and health outcomes; interrogating outcome-specific tissues or their carefully selected proxies; and conducting translational research that aims at dissecting the underlying mechanisms. Each is separately discussed below.

While most studies to date have associated life environments with epigenetic signatures at a single time point, there is pressing need for large-scale longitudinal studies that track how these signatures change over time. Such longitudinal studies may allow the temporal resolution of epigenetic changes as they emerge within individuals, thereby answering several critical questions: Which epigenetic sites change right after environmental stress and can thus be potential targets for early prevention? Which changes correlate with distinct outcomes and can possibly serve as disease biomarkers? How does genetic background predispose to biological embedding of certain environmental exposures in some individuals but not others? To minimize spurious findings, these studies should carefully define and document both life environments and health outcomes over time. Longitudinal studies that evaluate interventions could further tell us what may be the proper stage and time point to intervene with which treatment modality.

Future studies should also broaden their scope and shift from the traditional, disease-centered approach - that focuses on environmental risks and disease outcomes - to placing a stronger emphasis on positive life environments and health outcomes. This conceptual shift will allow us to identify not only epigenetic profiles that occur in stressful environments and predispose to worse health, but also those that emerge in nourishing environments and can promote health. Interventions that reproduce such 'positive' epigenetic changes could theoretically also impart health benefits. Today efforts to study these diverse end points can be accelerated by using smartphones and other digital devices that allow moment-by-moment quantification of behaviors and outcomes. Educating the public about the importance of their participation in such studies will greatly facilitate these efforts.

Because epigenetic changes are largely tissue-specific, it is of great value to examine, when possible, the tissue directly relevant for the health outcome of interest. For instance, the ability to examine resected tumors has in part accelerated the knowledge and applications of cancer epigenetics, whereas less progress has been made in 
other disease types such as brain disorders. Likewise, tissue accessibility is a bottleneck when examining how life environments drive epigenetic changes in yet healthy individuals. In all these cases, easily accessible tissues serve as proxies for the question of interest. Yet very few studies have determined the extent to which we can extrapolate epigenetic findings in proxy tissues, albeit promising findings exist [9]. Another approach to address this limitation is to image epigenetics in living humans. Such imaging modalities are still early in their development, but positron emission tomography was recently used to measure the levels of epigenetic modifiers in the living brain [10].

The ultimate task will be to dissect the molecular mechanisms underlying the associations we see in human populations. Such mechanistic dissection will require carefully designed experiments in cellular and animal model systems and a constructive crosstalk that will facilitate translation of findings between clinical and basic science. In particular, carefully designed experiments in cells can enhance understanding by mechanistically dissecting how the identified epigenetic sites become established and in turn shape tissue function. Such an approach has demonstrated, for example, that DNA methylation sites deregulated by a combined action of aging and stress may influence immune cell function, thus contributing to peripheral inflammation and disease risk [11]. Similar translational efforts can be pursued by using animal models, though the challenge in such efforts is that epigenetic sites are only partially conserved across species. Despite lack of conservation at individual sites, however, epigenetic phenomena may show conserved features that can be leveraged to enhance translation. For example, similar to our ability to estimate chronological age with DNA methylation arrays in humans [3], composite DNA methylationbased predictors of age have been developed in mice [12]. The predictive accuracy is comparable for both species, although the contributing DNA methylation sites are only partially conserved. Leveraging carefully selected model systems that can be experimentally manipulated can deepen our insights into epigenetic determinants of human health.

\section{Conclusion}

Our life encounters and ways in which we live have long been known to affect lifespan and health span. Recent advances in epigenetics have brought this old, intuitive knowledge under new light: What takes place in our lives can be embedded in our epigenomes, potentially influencing in profound ways the function of our genes, cells, and bodies. Moreover, these scientific advances bring about a shift in our way of thinking and a hopeful message: Biological embedding through the epigenome can be lasting but also preventable or even reversible, and gaining deeper insights into its workings may enable us to direct it for our benefit.

\section{Financial \& competing interests disclosure}

The authors have no relevant affiliations or financial involvement with any organization or entity with a financial interest in or financial conflict with the subject matter or materials discussed in the manuscript. This includes employment, consultancies, honoraria, stock ownership or options, expert testimony, grants or patents received or pending, or royalties.

No writing assistance was utilized in the production of this manuscript.

\section{References}

1. WHO. Noncommunicable diseases progress monitor (Licence: CC BY-NC-SA 3.0 IGO) (2017). https://apps.who.int/iris/bitstream/ha ndle/10665/258940/9789241513029-eng.pdf;jsessionid=22AEDACD8BCC6B1E702F558930610087?sequence=1

2. Danese A, Pariante CM, Caspi A, Taylor A, Poulton R. Childhood maltreatment predicts adult inflammation in a life-course study. Proc. Natl Acad. Sci. USA 104(4), 1319-1324 (2007).

3. Horvath S. DNA methylation age of human tissues and cell types. Genome Biol. 14(10), R115 (2013).

4. Elliott HR, Tillin T, McArdle WL et al. Differences in smoking associated DNA methylation patterns in South Asians and Europeans. Clin. Epigenet.6(1), 4 (2014).

5. Liu C, Marioni RE, Hedman Å et al. A DNA methylation biomarker of alcohol consumption. Mol. Psych. 23(2), 422-433 (2018).

6. Zannas AS, Arloth J, Carrillo-Roa T et al. Lifetime stress accelerates epigenetic aging in an urban, African American cohort: relevance of glucocorticoid signaling. Genome Biol. 16(1), 266 (2015).

7. Fraga MF, Ballestar E, Paz MF et al. Epigenetic differences arise during the lifetime of monozygotic twins. Proc. Natl Acad. Sci. USA 102(30), 10604-10609 (2005).

8. Dawson MA. The cancer epigenome: concepts, challenges, and therapeutic opportunities. Science NY355(6330), 1147-1152 (2017).

9. Stueve TR, Li WQ, Shi J et al. Epigenome-wide analysis of DNA methylation in lung tissue shows concordance with blood studies and identifies tobacco smoke-inducible enhancers. Hum. Mol. Genet. 26(15), 3014-3027 (2017). 
10. Wey HY, Gilbert TM, Zurcher NR et al. Insights into neuroepigenetics through human histone deacetylase PET imaging. Sci. Translat. Med. 8(351), 351ra106 (2016).

11. Zannas AS, Jia M, Hafner K et al. Epigenetic upregulation of FKBP5 by aging and stress contributes to NF-kappaB-driven inflammation and cardiovascular risk. Proc. Natl Acad. Sci. USA doi:10.1073/pnas.1816847116 (2019) (Epub ahead of print).

12. Stubbs TM, Bonder MJ, Stark AK et al. Multi-tissue DNA methylation age predictor in mouse. Genome Biol.18(1), 68 (2017). 